\title{
Signal processing issues for the exploitation of pulse-to-pulse encoding SAR transponders
}

Merryman Boncori, John Peter; Schiavon, Giovanni

Published in:

I E E E Transactions on Geoscience and Remote Sensing

Link to article, DOI:

10.1109/TGRS.2007.910714

Publication date:

2008

Document Version

Publisher's PDF, also known as Version of record

Link back to DTU Orbit

Citation $(A P A)$ :

Merryman Boncori, J. P., \& Schiavon, G. (2008). Signal processing issues for the exploitation of pulse-to-pulse encoding SAR transponders. I E E E Transactions on Geoscience and Remote Sensing, 46(4), 1048-1057. https://doi.org/10.1109/TGRS.2007.910714

\section{General rights}

Copyright and moral rights for the publications made accessible in the public portal are retained by the authors and/or other copyright owners and it is a condition of accessing publications that users recognise and abide by the legal requirements associated with these rights.

- Users may download and print one copy of any publication from the public portal for the purpose of private study or research.

- You may not further distribute the material or use it for any profit-making activity or commercial gain

- You may freely distribute the URL identifying the publication in the public portal 


\title{
Signal Processing Issues for the Exploitation of Pulse-to-Pulse Encoding SAR Transponders
}

\author{
John Peter Merryman Boncori, Member, IEEE, and Giovanni Schiavon, Member, IEEE
}

\begin{abstract}
Synthetic aperture radar signal processing issues related to the exploitation of a pulse-to-pulse encoding transponder using pseudorandom codes discussed analytically. Namely the focusing algorithm, the code synchronization procedure and the properties of the code induced gain against non-encoding point scatterers and distributed ones. A time-domain processing algorithm and a code synchronization procedure are proposed and validated on simulated data and on a European Remote Sensing Satellite-2 data set containing prototypes of such a device. The interaction of the transponder signal with terrain backscattering is analyzed by deriving parameters that are useful for performance assessment. These are related to the relevant parameters in radiometric calibration, interferometric applications, and tagging.
\end{abstract}

Index Terms-Azimuth phase coding, synthetic aperture radar (SAR) signal processing, SAR transponder, time-domain correlation (TDC).

\section{INTRODUCTION}

$\mathbf{S}$ YNTHETIC aperture radar (SAR) transponders have mostly received interest for sensor external calibration [1]-[3]. Due to their limited size and weight, they may be more cost effective and environmentally friendly than corner reflectors. Furthermore, modulation techniques have been proposed to decouple the transponder signal from the backscattering of its surroundings [2], [4]-[6]. Other applications have also been suggested in the literature, such as interferometry [7] and tagging [4], [8]. Interest in these applications is increased by forthcoming X-band SAR systems and constellations.

This paper concerns a pulse-to-pulse modulating transponder. A block diagram summarizing the architecture proposed in [4] is given in Fig. 1. While within the azimuth beamwidth of the sensor, the device is capable of receiving a sequence of SAR pulses and superimposing a code sequence prior to retransmission toward the radar. Encoding is accomplished by binary phase-shift keying (BPSK) modulation on each received pulse. When the code sequence applied by the device is used during data processing to focus the transponder, the SAR processor filter is no longer matched to the electric field backscattered from nonencoding point scatterers, thus providing a means for localizing and identifying the encoding transponder in the SAR image.

Manuscript received January 4, 2006; revised May 23, 2007.

J. P. Merryman Boncori is with the Danish National Space Center (DRC), Denmark's Technical University, 2800 Lyngby, Denmark (e-mail: jme@space.dtu.dk).

G. Schiavon is with the Dipartimento di Informatica, Sistemi e Produzione, University of Rome "Tor Vergata," 00133 Rome, Italy (e-mail: schiavon@ disp.uniroma2.it).

Digital Object Identifier 10.1109/TGRS.2007.910714

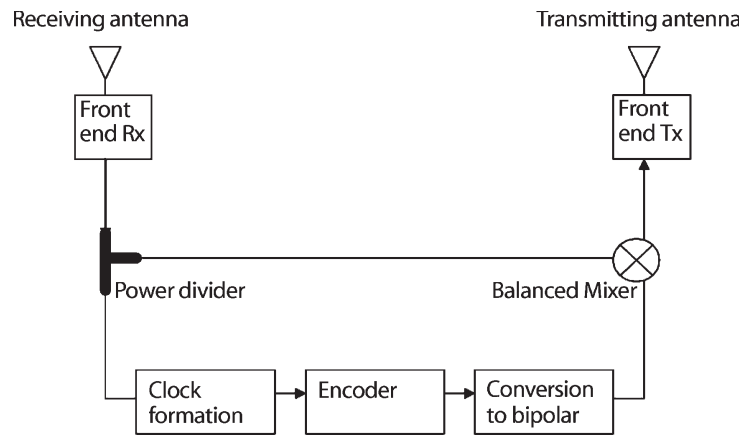

Fig. 1. Block diagram of the pulse-to-pulse encoding SAR transponder proposed in [4]. Each received SAR pulse is amplified in the receiver front end and split onto two branches through a power divider. In the bottom branch, a modulating signal is derived. The clock formation block generates a triggering signal for the subsequent binary (pseudorandom) encoder. Binary values are converted to a bipolar signal ( $\pm V$, where $V$ is a constant voltage), which is then multiplied with the input radar pulse in a balanced mixer, accomplishing BPSK modulation through an amplitude modulation. The modulated pulse is filtered and amplified in the transmission front end prior to retransmission toward the radar.

The objective of this paper is twofold. First, a fully automated method of implementing the matched filter required to image the device is described in detail. Second, the properties and interaction of the matched and mismatched signals are analyzed in order to assess the benefits of the considered co/decoding method for the previously mentioned applications.

The analytical models for subsequent discussions are presented in Section II. A focusing algorithm based on azimuth time-domain correlation (TDC) is described in Section III. Simulated data are used in Section IV to validate the proposed algorithm and analyze the properties of focused and defocused point scatterers when imaged through the algorithm of Section III. The interaction of the transponder signal with terrain backscattering is discussed in Section V, defining parameters for performance assessment. The derived focusing algorithm and the interaction analysis findings are demonstrated on a real data set in Section VI. Their application to radiometric calibration, interferometry, and tagging is discussed in Section VII, and finally, conclusions are drawn in Section VIII.

\section{Problem Statement}

\section{A. Signal Impulse Response}

The convolutional model shown in Fig. 2 is often used to represent a coherent SAR system [9]. Coordinates $\tau$ and $\eta$ represent respectively range (pulse propagation) time and azimuth (satellite motion) time elapsed from the zero Doppler 


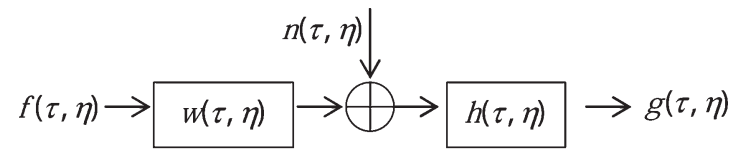

Fig. 2. Block diagram of a single-look SAR system.

point. The system output $g(\tau, \eta)$ represents the complex SAR image and is given by

$g(\tau, \eta)=f(\tau, \eta) \otimes w(\tau, \eta) \otimes h(\tau, \eta)+n(\tau, \eta) \otimes h(\tau, \eta)$

where $\otimes$ is the convolution operator; $w(\tau, \eta)$ and $h(\tau, \eta)$ are the SAR system and processor impulse responses, respectively; and $n(\tau, \eta)$ is the receiver's thermal noise. The input $f(\tau, \eta)$ is such that $f(\tau, \eta) \otimes w(\tau, \eta)$ represents the complex electric field at the radar backscattered from a resolution cell. The following expression holds [10]:

$$
\begin{aligned}
& w(\tau, \eta)=p_{r}(\tau-2 R(\eta) / c) \cdot p_{a}\left(\eta-\eta_{c}\right) \\
& \quad \cdot \exp \{-j 4 \pi R(\eta) / \lambda\} \cdot \exp \left\{j \pi K_{r}(\tau-2 R(\eta) / c)^{2}\right\}
\end{aligned}
$$

where $p_{r}(\cdot)$ and $p_{a}(\cdot)$ represent the transmitted pulse's envelope and the azimuth antenna pattern, respectively, whereas $R, K_{r}$, and $\eta_{c}$ denote the slant range, transmitted chirp rate, and beam center time.

When a point scatterer is imaged, $f(\tau, \eta)$ is proportional to a Dirac function, so that its backscattered electric field will be observed over a certain time span in both dimensions and be proportional to $w(\tau, \eta) . w(\tau, \eta)$ will be characterized by the phase modulation given to the transmitted radar pulse in the range time dimension and by the Doppler effect phase modulation in the azimuth time dimension. Modeling $n(\tau, \eta)$ as a Gaussian white noise process, the processor, which maximizes the signal-to-noise ratio is known to be the matched filter

$$
h(\tau, \eta)=w^{*}(-\tau,-\eta)
$$

Considering now the problem of imaging a pulse-to-pulse encoding transponder, its previously outlined working principle produces a sequence of alterations in the hyperbolic Doppler phase modulation. From this point of view, everything goes as if the device were a nonencoding point scatterer imaged by a radar with the modified impulse response, i.e.,

$$
w_{\text {code }}(\tau, \eta)=w(\tau, \eta) \cdot \exp \{j \theta(\eta)\}
$$

where function $\theta(\eta)$ represents the sequence of phase alterations due to the transponder's internal encoder and ideally takes on only values 0 and $\pi$. To achieve azimuth focusing, i.e., for all samples to coherently add in voltage in this dimension, compensation of both Doppler and code-induced phase modulation is required. The suitable processor can again be considered the matched filter

$$
h_{\text {code }}(\tau, \eta)=w_{\text {code }}^{*}(-\tau,-\eta) .
$$

\section{B. Azimuth Frequency Domain}

In conventional processing, compensation for Doppler modulation can be carried out in the Doppler frequency domain since time and frequency are locked together due to the fact that, for each frequency, a single point of stationary phase exists. For the imaging of an encoding transponder, this principle cannot be exploited though. In fact, the modulating sequence superimposed by the device's encoder in (4) can be considered as an amplitude term, which is a sequence of pseudorandom sign changes, multiplying the linear frequency-modulated waveform in (2). In taking now the Fourier transform of (4) in the azimuth dimension, contributions to the Fourier integral of positive and negative loops of the oscillating function $\exp \{-j 4 \pi R(\eta) / \lambda\}$ do not cancel due to the code-induced amplitude term, which rapidly varies with respect to angle changes. This implies that code-induced phase modulations must be compensated for in the azimuth time domain.

\section{Code Synchronization}

Removal of the phase alterations produced by the device also requires knowledge of which is the first SAR pulse to trigger the transponder's internal encoder and be backscattered with enough power to be detected by the SAR receiver. This, in turn, determines the azimuth position in the SAR raw data at which the sequence of phase alterations starts.

In this paper, we will suppose that this information is not available, although we will assume in the next section that pseudorandom codes with good autocorrelation properties are used, so that these can be exploited in the code acquisition procedure, as in mobile communication systems and global positioning systems.

It must be pointed out that a code synchronization procedure could be avoided or anyway simplified by providing a time stamp for the first received pulse, as done, for example, by the device presented in [11]. This may however not be practical for all applications, and anyhow, a method to recover this information from the data can be useful in case of time-stamping failures.

In the following, it will finally be supposed that the device's sensitivity is given such that the encoding starts before the integration time, i.e., a pulse transmitted outside the $-6-\mathrm{dB}$ (two-way) azimuth antenna pattern triggers the transponder's encoder. This is an easily met specification for current SAR systems and is also required for full exploitation of the device. Therefore, it could be considered as a technical specification rather than an assumption.

\section{Modified Time-Domain Correlation}

A conceptually straightforward solution proposed for the focusing of a pulse-to-pulse encoding transponder [12] consists of modifying the conventional TDC azimuth focusing algorithm [13]. To avoid confusion, we point out that the TDC terminology has also been used in the literature to denote an exact 2-D processing algorithm, although, in this paper, it will be used to refer to an azimuth compression technique only. 


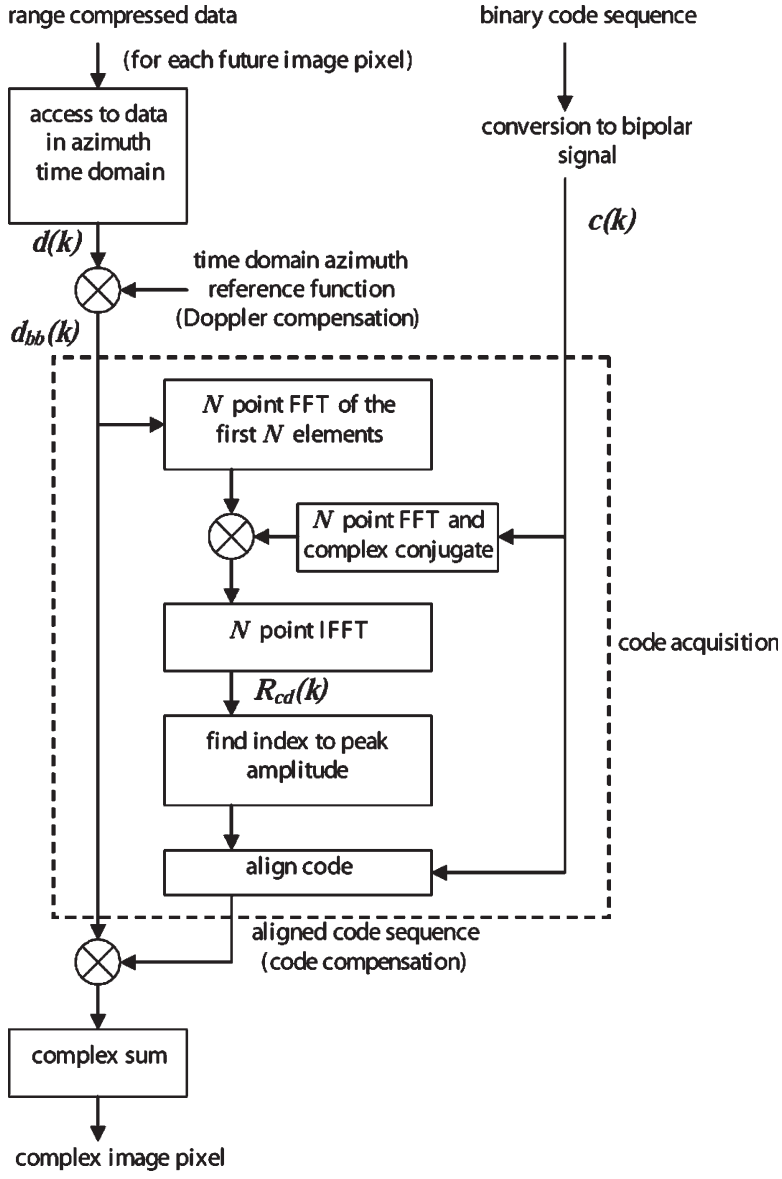

Fig. 3. Block diagram of the proposed processing algorithm (single-look image).

A block diagram of the proposed processing algorithm, with an improved code synchronization strategy compared to [12], is shown in Fig. 3. Range compression can be carried out in the frequency domain since the encoding method does not affect the signal characteristics in this dimension. Azimuth focusing is based on the time-domain compensation of Doppler and code-induced phase modulations. The correct code alignment is obtained by searching for the index to the peak amplitude of the code's autocorrelation function.

Following range compression, denoting the length of the code word used by the device as $N$, an $N$-point DFT of the known code word $c(k)$ is taken, and each resulting element is complex conjugate and stored. For each future image pixel, data are then retrieved from the range migration locus in the azimuth time domain by interpolating adjacent samples in the slant range dimension, obtaining sequence $d(k)$. Compensation of the Doppler phase modulation is then carried out by multiplication with the conventional azimuth time-domain reference function, obtaining sequence $d_{b b}(k)$. The appropriate code alignment is searched for by computing the cross correlation $R_{c d}(k)$ between $c(k)$ and the first $N$ elements of $d_{b b}(k)$. This can be done by computing the circular convolution between $d_{b b}(k)$ and $c^{*}(-k)$, using DFTs. If $d(k)$ contained only the range-compressed data of an encoding transponder, the result of the circular convolution would be proportional to the code's autocorrelation function $R_{c}(k)$, which is centered on the code
TABLE I

SimUlation PARAMETERS

\begin{tabular}{l|l}
\hline \multicolumn{1}{c|}{ Parameter } & \multicolumn{1}{c}{ Value } \\
\hline Incidence angle & $23^{\circ}$ \\
Slant range & $847 \mathrm{~km}$ \\
Doppler bandwidth & $1378 \mathrm{~Hz}$ \\
PRF & $1679.9 \mathrm{~Hz}$ \\
Doppler centroid & $0 \mathrm{~Hz}$ \\
Azimuth FM rate & $-2100 \mathrm{~Hz} / \mathrm{s}$ \\
Number of integrated pulses & 1101 \\
Gold code length & $31,127,255,511,1023$ \\
\hline
\end{tabular}

shift between data $d_{b b}(k)$ and reference $c(k)$. If $R_{c}(k)$ has a sharp peak in the origin, the index to its maximum amplitude can be found, and this will correspond to the sought code shift. The code-induced phase modulation is then compensated for using the appropriate alignment. Finally, the compensated data is summed, yielding the complex pixel value.

\section{TRAnSPONDER Signal PROPERTIES}

A point scatterer simulator was first used to verify the focusing algorithm presented in the previous section and analyze the properties of the focused and defocused transponder signals. Gold code words of different lengths were chosen. These sequences have pseudorandom properties and low crosscorrelation values, for which they are widely used in codedivision multiple access mobile communication systems [14]. They are generated using two linear feedback shift registers, with feedback connections specified by so-called "preferred pairs" of characteristic polynomials. Those tabulated in [15] were used in this paper. A shift register with $n$ taps will generate a code word of length $N=2^{n}-1$.

Trials were also carried out with pseudonoise (PN) sequences [14]. The results obtained however differ only very slightly from those obtained with Gold codes and will be mentioned only in the conclusions.

\section{A. Characteristics of the Focused Signal}

The raw data of an encoding transponder were simulated using (2) and (3). The processing algorithm described in Fig. 3 was then used to focus the data. In order to allow a comparison with a real data set (Section VI), the SAR parameters of the European Space Agency's European Remote Sensing Satellite-2 (ERS-2) mission were used in the simulations, as shown in Table I. The results are presented for a zero squint angle, although it has been verified that, as theoretically expected, this parameter has no impact on the processing algorithm. No thermal noise or scattering from the surrounding was simulated at this stage.

Examples of simulated encoding transponder images are shown in Fig. 4, as a result of conventional and modified TDCs, respectively. An increase in the expected integrated sidelobe ratio (ISLR) of each focused point scatterer is apparent. Its signal is nonnull over the entire length of azimuth convolution 


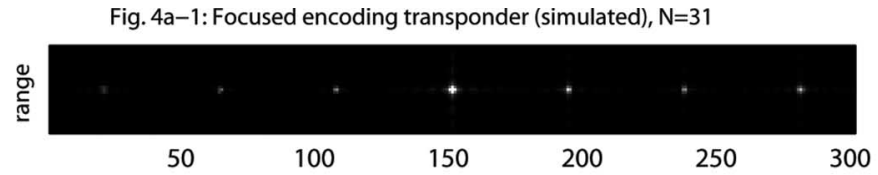

Fig. 4a-2: Focused encoding transponder (simulated), $\mathrm{N}=255$

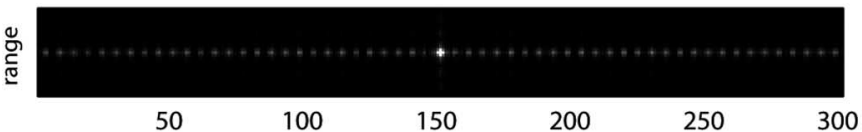

Fig. 4a-3: Focused encoding transponder (simulated), $\mathrm{N}=1023$

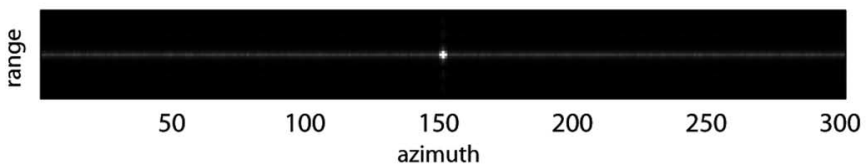

(a)

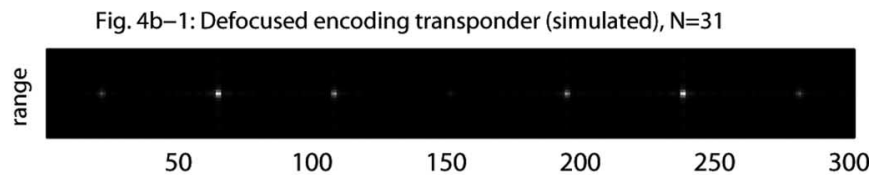

Fig. 4b-2: Defocused encoding transponder (simulated), $\mathrm{N}=255$

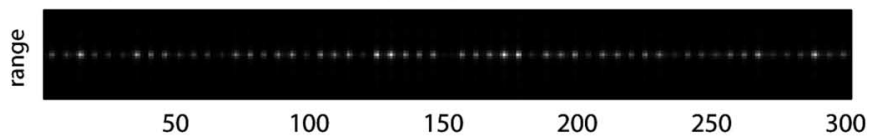

Fig. 4b-3: Defocused encoding transponder (simulated), $\mathrm{N}=1023$

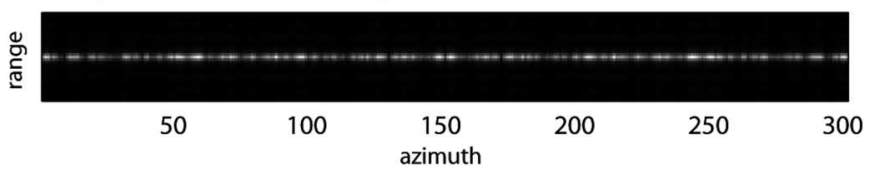

(b)

Fig. 4. (a) Focused and (b) defocused simulated encoding transponders, using Gold code words of different lengths.

TABLE II

Simulated Point TARget AZIMUTh IRF PARAMETERS AFTER MATCHED FILTERING

\begin{tabular}{c|c|c|c}
\hline & $\begin{array}{c}-3 \text { dB width } \\
(\text { lines })\end{array}$ & $\begin{array}{c}\text { PSLR } \\
(\mathrm{dB})\end{array}$ & $\begin{array}{c}\text { ISLR } \\
(\mathrm{dB})\end{array}$ \\
\hline non encoded & 1.28 & -20.84 & -18.30 \\
\hline $\begin{array}{c}\text { encoded } \\
n=5\end{array}$ & 1.28 & -8.57 & 2.04 \\
$n=7$ & 1.28 & -15.55 & 3.54 \\
$n=8$ & 1.28 & -16.63 & 4.28 \\
$n=9$ & 1.28 & -17.12 & 5.25 \\
$n=10$ & 1.28 & -17.26 & 7.16 \\
\hline
\end{tabular}

( $2 L_{\mathrm{az}}-1$ samples, where $L_{\mathrm{az}}$ is the azimuth reference function length). This is due to the fact that the synchronization procedure of Section III fails for the lower level sidelobes, which are not properly compensated for the code-induced modulation, appearing defocused in the final image. This fact however does not compromise the retrieval of the peak amplitude and phase, for which code alignment is successful and the encoder's phase modulation can be properly compensated for by the algorithm of Section III. Other relevant azimuth impulse response function (IRF) parameters are reported in Table II. No significant loss of resolution was observed, compared to conventional processing.

Recovery of the conventional azimuth IRF is possible, although it would require a second processing run. In fact, once the correct code alignment is known, the image can be reprocessed, appropriately compensating each pixel of the point target response for the code-induced modulation.

\section{B. Characteristics of the Defocused Signal}

From Fig. 4(b), it can be seen that the defocused signal of a nonencoding point target processed according to the algorithm of Section III appears to be composed of a number of equally spaced peaks with a code-length-dependent spacing. This was also observed by other researchers [16], although no explanation was given. This effect is caused by the phase structure of the signal, which appears in the azimuth convolution. Placing the azimuth time origin at the beam center point, the $k$ th point of the convolution from the origin, considering only the $k>0$ case, is given for a zero squint angle by

$$
\begin{aligned}
& \sum_{m=1}^{L_{\mathrm{az}}-k}\left(\exp \left[j \pi f_{R}\left(\frac{m}{\mathrm{PRF}}\right)^{2}\right]\right. \cdot \exp [j \theta(m)] \\
&\left.\cdot \exp \left[-j \pi f_{R}\left(\frac{k+m}{\mathrm{PRF}}\right)^{2}\right]\right) .
\end{aligned}
$$

In (6), $f_{R}$ and PRF represent the azimuth frequency modulation (FM) rate and the radar pulse repetition frequency, respectively, and $\theta(m)$ represents the sequence of phase alterations used in the phase compensation step of Fig. 3. Considering the product of a linear FM signal and a delayed version of its complex conjugate in (6), for different values of the delay $k$, the phase across the vector that was obtained as $m$ varies through its allowed values will take a different number of samples to exhibit a $360^{\circ}$ rotation. For example, using ERS SAR parameters, for $k=44$, the chirp product in (6) takes 31 samples to complete a $360^{\circ}$ variation. For $k=6,255$ samples are required. This implies that, if this chirp product is multiplied by an encoding sequence with period 31 or 255 , respectively, as done in the algorithm of Section III, a certain number of samples will coherently add up in (6), giving rise to a peak in the output. Moreover, this is true regardless of the initial code shift of the shift register and of the specific code word used. Therefore, in general, about $\left(2 L_{\mathrm{az}}-1\right) / k$ peaks are expected for the dispersed signal of a nonencoding point scatterer. Their amplitude will decrease, moving away from the position of the scatterer since less in-phase elements are summed in (5) for increasing values of $k$.

\section{Transponder Performance in a Random Scene}

For the applications, it is necessary to quantify the interactions between nonencoded and phase-encoded signals. In particular, for external calibration and interferometry, it is of interest to know whether the co/decoding method provides a processing gain in terms of signal-to-clutter $(\mathrm{S} / \mathrm{C})$ ratio and, thus, an increased decoupling between the point scatterer of interest and the surrounding environment. For tagging 
applications, the minimum detectable radar cross section (RCS) as a function of radar parameters, code properties, and environment backscattering is to be determined.

In this section, the parameters required to assess transponder performance are derived. Their impact on the applications will be discussed in Section VII.

\section{A. Signal Decoupling}

Supposing that an encoding transponder is deployed, the input signal to the convolutional model of Fig. 2 will be

$$
f(\tau, \eta)=\sqrt{\sigma} \cdot \delta(\tau, \eta)+c(\tau, \eta) .
$$

The deterministic part, which represents the transponder, is a Dirac function with amplitude $\sqrt{\sigma}$, where $\sigma$ is the RCS of the device, whereas $c(\tau, \eta)$ is, in general, a nonstationary circular Gaussian white noise process modeling terrain scattering.

To focus the encoding transponder, the algorithm described in Section III can be used. It will be assumed in the following that the code alignment is already known since the outlined synchronization procedure can be used to recover it under the conditions derived in the next section. In this way, all pixels are compensated for the same code-induced phase modulation, the one suitable to focus the encoding device.

To derive the processing gains, the output of the processor can be written based on Fig. 2 as

$$
\begin{aligned}
g(\tau, \eta)= & \left(\sqrt{\sigma} \cdot \delta(\tau, \eta) \otimes w_{\text {code }}(\tau, \eta)\right) \otimes h_{\text {code }}(\tau, \eta) \\
& +(c(\tau, \eta) \otimes w(\tau, \eta)+n(\tau, \eta)) \otimes h_{\text {code }}(\tau, \eta)
\end{aligned}
$$

where it has been considered, as detailed in Section II, that everything goes as if the encoding point scatterer experienced an altered Doppler history with respect to natural ones. Parameter $G_{d}$ may be defined as the increase in peak transponder signal intensity to mean terrain backscattering power due to the co/decoding method. Following a procedure similar to the one used in [9], its expected value can be proven to be

$$
E\left[G_{d}\right]=\frac{\int|w(\tau, \eta) \otimes h(\tau, \eta)|^{2} d \tau d \eta}{\int\left|w(\tau, \eta) \otimes h_{\text {code }}(\tau, \eta)\right|^{2} d \tau d \eta} .
$$

The numerator in (9) represents the integral of the squared modulus of the conventional 2-D IRF, whereas the denominator integrand is the squared modulus of the dispersed signal, which appeared in Fig. 4(b).

For completeness, it is worth quantifying the effect of the encoding method on a nonencoded point scatterer signal. Gain $G_{p}$ can be defined as the ratio of the peak encoding transponder intensity $P_{\text {peak } \_c}$ to the power of a nonencoding point scatter with the same RCS, which was processed with the use of a code sequence. Denoting the spatial average operator with \langle\rangle , the following parameters of interest can be derived:

$$
\begin{aligned}
E\left[G_{p}\right] & =\frac{P_{\text {peak_c }}}{\left\langle\left|w(\tau, \eta) \otimes h_{\text {code }}(\tau, \eta)\right|^{2}\right\rangle} \\
\min G_{p} & =\frac{P_{\text {peak_c }}}{\max \left|w(\tau, \eta) \otimes h_{\text {code }}(\tau, \eta)\right|^{2}} .
\end{aligned}
$$

TABLE III

Processing GaINS (IN DECIBELS) COMPUTED FOR 1000 DIFFERENT GOLD CODE WORDS

\begin{tabular}{l|cc|cc|cc|cc|cc}
\hline$n$ & \multicolumn{2}{|c|}{$E\left[\mathrm{G}_{\mathrm{d}}\right]$} & \multicolumn{2}{|c|}{$\left\langle\mathrm{G}_{\mathrm{p}}>\right.$} & \multicolumn{2}{|c|}{$\min \mathrm{G}_{\mathrm{p}}$} & \multicolumn{2}{|c|}{$\left\langle\mathrm{G}_{\mathrm{c}}\right\rangle$} & \multicolumn{2}{|c}{$\min \mathrm{G}_{\mathrm{c}}$} \\
& mean & std & mean & std & mean & std & mean & std & mean & std \\
\hline 5 & 1.17 & 0.23 & 33.40 & 0.23 & 11.59 & 1.47 & 33.42 & 0.01 & 15.51 & 0.02 \\
7 & 1.15 & 0.11 & 33.38 & 0.11 & 16.24 & 1.16 & 33.39 & 0.01 & 21.10 & 0.11 \\
8 & 1.15 & 0.08 & 33.38 & 0.08 & 18.44 & 0.96 & 33.38 & 0.01 & 23.89 & 0.13 \\
9 & 1.15 & 0.07 & 33.38 & 0.07 & 20.79 & 0.79 & 33.38 & 0.02 & 26.35 & 0.17 \\
10 & 1.15 & 0.08 & 33.38 & 0.08 & 22.21 & 0.81 & 33.38 & 0.06 & 26.08 & 0.24 \\
\hline
\end{tabular}

Similarly, gain $G_{c}$ compared to an encoding point target using a different code is given by

$$
\begin{aligned}
E\left[G_{c}\right] & =\frac{P_{\text {peak_c1 }}}{\left\langle\left|w_{\text {code2 } 2}(\tau, \eta) \otimes h_{\text {code1 } 1}(\tau, \eta)\right|^{2}\right\rangle} \\
\min G_{c} & =\frac{P_{\text {peak_c1 }}}{\max \left|w_{\text {code2 } 2}(\tau, \eta) \otimes h_{\text {code1 } 1}(\tau, \eta)\right|^{2}} .
\end{aligned}
$$

The processing gains (9)-(11) can be numerically computed using the 2-D SAR system and processor impulse responses (2)-(5). In the defining expressions for the processing gains, convolution with $h(\tau, \eta)$ represents conventional TDC processing, whereas convolution with $h_{\text {code }}(\tau, \eta)$ implies applying the modified algorithm described in Section III, where the synchronization procedure is skipped though and the right code alignment is directly used for phase compensation. Simulations over 1000 different Gold code words yielded the mean values and the standard deviations reported in Table III. Similar values for $G_{d}$ and $G_{p}$ were reported in [16] and [17], respectively. A qualitative explanation to the different orders of magnitude of these two gains can be given, considering each resolution cell containing backscattering terrain as a point scatterer and considering the defocusing of a point scatterer due to the encoding method. The average point scatterer signal suppression in azimuth is about $33.4 \mathrm{~dB}$, and it is not null over $2 L_{\mathrm{az}}-1$ azimuth samples. The dispersed signals of adjacent resolution azimuth cells incoherently add up with an intensity gain of about $2 L_{\mathrm{az}}-1$, in which $L_{\mathrm{az}}$ is on the order of 1000 . The overall gain is thus almost null.

From Table III, it can also be seen that the S/C ratio obtained, and thus the decoupling between the transponder signal and the backscattered field from the surroundings, is code length independent. On the contrary, the gains compared to a nonencoding point target or to an encoding one using a different code improve with increasing code length.

\section{B. Code Synchronization}

The success of the synchronization procedure described in Section III relies on the device's RCS and on the shape of the code autocorrelation function, which improves as the code length increases. On the other hand in order to improve 
TABLE IV

SyNCHRONIZATION MARGIN (IN DECIBELS) FOR DIFFERENT GOLD CODE LENGTHS

\begin{tabular}{c|cc|cc|cc|cc|cc}
\hline $\begin{array}{l}\sigma / \sigma^{0} \\
(\mathrm{~dB})\end{array}$ & \multicolumn{2}{|c|}{$n=5$} & \multicolumn{2}{|c|}{$n=7$} & \multicolumn{2}{|c|}{$n=8$} & \multicolumn{2}{c|}{$n=9$} & \multicolumn{2}{c}{$n=10$} \\
\hline & mean & std & mean & std & mean & std & mean & std & mean & std \\
40 & - & - & - & - & - & - & 6.67 & 1.25 & 9.41 & 0.92 \\
45 & - & - & - & - & 6.98 & 1.38 & 10.93 & 0.85 & 13.71 & 0.83 \\
50 & - & - & 7.65 & 1.28 & 10.75 & 0.95 & 14.39 & 0.70 & 17.1 & 0.57 \\
55 & 5.42 & 1.38 & 10.69 & 1.00 & 13.74 & 0.84 & 17.22 & 0.67 & 19.31 & 0.53 \\
60 & 7.49 & 1.08 & 13.11 & 0.73 & 15.23 & 0.64 & 18.92 & 0.48 & 20.41 & 0.44 \\
\hline
\end{tabular}

processing efficiency it would be desirable to choose short code lengths. Furthermore, the code acquisition strategy will be shown to affect the peak-to-background ratio (PBR) of the device in the resulting image. The aim of this section is to quantify the existing tradeoffs between code length and device RCS for design purposes. Stationarity of the scene statistics will be assumed in this section, whereas the performance in a nonhomogeneous scene will be discussed in the following one.

A distributed raw data simulator was implemented, and an encoding transponder signal with a known RCS was placed in a homogeneous scene, i.e., pure speckle, with a known $\sigma^{0}$. Thermal noise was modeled by an additive Gaussian white noise process with a $-21-\mathrm{dB}$ noise-equivalent $\sigma^{0}$. Gold codes of different lengths were used to provide an azimuth phase encoding in the raw data and, subsequently, during the processing. For each code, the processing algorithm described in Section III was used to assess the performance of the synchronization procedure by computing a parameter that will hereafter be referred to as the synchronization margin (SM). It represents the ratio between the two greatest peaks of the synchronization signal $R_{c d}(k)$ (see Fig. 3 and its description in the text).

The mean observed values for the SM are reported in Table IV, together with their standard deviations. These values depend on code length $N=2^{n}-1$ and on the $\sigma / \sigma^{0}$ ratio. For certain combinations of these parameters, no values are reported in Table IV, because the peak position of $R_{c d}(k)$ was incorrectly determined in more than $1 \%$ of the trials. The procedure was instead always successful for the combinations corresponding to the reported values.

A lower bound for the SM can be theoretically obtained for a code family with known autocorrelation properties and arbitrary $\sigma / \sigma^{0}$. It can be proven that

$$
\mathrm{SM}=\frac{\left|2^{n}-1+\sqrt{\min \mathrm{CG}(n)} \sqrt{\left(\sigma^{0} \Delta x \Delta R_{g}\right) /\left(\sigma L_{r} W_{r}\right)}\right|^{2}}{\left|t(n)+\sqrt{\operatorname{maxCG}(n)} \sqrt{\left(\sigma^{0} \Delta x \Delta R_{g}\right) /\left(\sigma L_{r} W_{r}\right)}\right|^{2}} .
$$

In (12), $L_{r}$ stands for the range reference function length, $W_{r}$ is the loss in peak signal strength due to the range weighting function (e.g., Kaiser weighting), $t(n)$ is the maximum value of the code autocorrelation function in a point different from the origin, and $\Delta x$ and $\Delta R_{g}$ are the unprocessed azimuth and
TABLE V

Average PBR Loss Due to the Code Acquisition Procedure

\begin{tabular}{c|c}
\hline$n$ & PBR loss $(\mathrm{dB})$ \\
\hline 5 & 0.41 \\
7 & 1.62 \\
8 & 3.06 \\
9 & 5.79 \\
10 & 8.60 \\
\hline
\end{tabular}

ground range resolutions, respectively. The latter resolutions are given by $\Delta x=\lambda R / L_{a}$ and $\Delta R_{g}=c \tau /(2 \sin \theta)$, respectively, where $L_{a}$ is the SAR along-track antenna length and $\tau$ is the transmitted pulse duration. For Gold codes, $t(n)$ is instead given by [14]

$$
t(n)= \begin{cases}2^{\frac{n+1}{2}}+1, & \text { for } n \text { odd } \\ 2^{\frac{n+2}{2}}-1, & \text { for } n \text { even. }\end{cases}
$$

Parameter $\mathrm{CG}(n)$, which stands for "correlation gain" and is a function of $n$, represents the intensity gain of the signal backscattered from the terrain after correlation with the code sequence. The minimum and maximum values have been numerically computed. A vector of $N$ independent random variables having uniform phase and Rayleigh amplitude distributions was correlated with a binary code sequence converted to a bipolar signal (using a $1 \rightarrow-1$ and $0 \rightarrow 1$ correspondence). The intensity gain compared to the random vector's expected intensity value was computed. This was repeated for 1000 random vectors and different code words. The following relations were found to fit the simulated data with sufficient accuracy:

$$
\begin{aligned}
\max \mathrm{CG}(n) \cong n\left(2^{n}-1\right) \\
\min \mathrm{CG}(n) \cong 1 .
\end{aligned}
$$

It is finally reported that the code acquisition procedure was found to worsen the PBR compared to the case of a code alignment known a priori. The observed PBR loss proved to be code length dependent, as reported in Table V. In particular, it was verified to be independent of the $\sigma / \sigma^{0}$ ratio. PBR loss is due to the fact that the procedure of Section III not only correctly identifies the right phase compensation for a pixel containing an encoding transponder but also maximizes the nonencoded signals in the resulting image over all possible code shifts. This undesired optimization leads to significant results due to the high variability of $\mathrm{CG}(n)$ for a fixed $n$. The code length dependence of the PBR loss may be imputed to the fact that the excursion of the azimuth convolution (6) over all possible code shifts decreases with code length. We believe this to be due to a property of pseudorandom codes, for which shorter code words contain shorter runs of ones and, therefore, of sign changes [14]. This, in turn, causes the sum in (6) to become less sensitive to code shifts as code length decreases.

\section{Effects of a Nonhomogenoeus Random Scene}

As far as decoupling is concerned, it is first pointed out that no assumption on the stationarity of the scene statistics, i.e., scene homogeneity, is needed to derive (9), which represents the expected gain in terms of S/C. In a nonhomogeneous scene, 


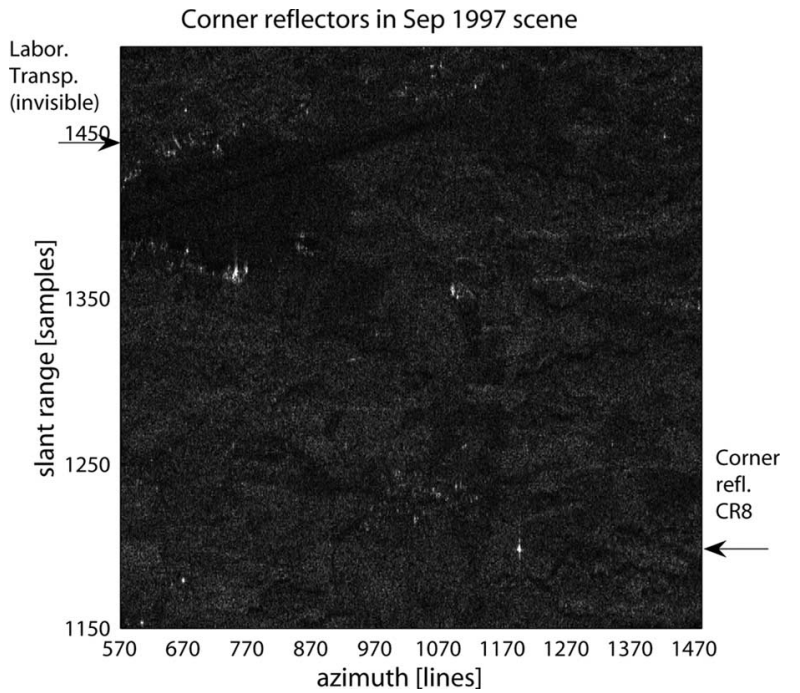

Fig. 5. Corner reflectors in the scene from September 1997 (conventional processing).

fluctuations around this expected value will be greater than in a homogeneous one. In fact, from the description of the defocused signal characteristics in Section IV-B, it is expected that, in a nonhomogeneous environment, the value of the S/C decoupling will be specific to the imaged scene and depend on the average intensity of scatterers that are located at the same slant range cell, as well as those spanned by the range cell migration locus, and within a synthetic aperture of the transponder position in azimuth. As far as SM is concerned, the results obtained in the previous section, including (12), are expected to also hold in the nonhomogeneous case, substituting $\sigma^{0}$ with its spatial average.

\section{Real Data PRocessing}

Two data sets from encoding transponder experiments performed by the Microwave and Radar Institute (HR) of the German Aerospace Center (DLR) in 1997/1998 [17] were investigated. In both scenes, prototypes of the encoding transponder described in [4] are deployed, as well as reference nonencoding corner reflectors of known position and RCS, as shown in Figs. 5 and 6. In Fig. 5, the encoding transponder is not visible, its defocused signal being below the backscattering intensity level from the surroundings. The two encoding devices appear instead as horizontal streaks in Fig. 6 after conventional processing, due to their large RCSs. The Uncoded and Code4 transponders were deployed in grassland, about $100 \mathrm{~m}$ away from buildings in the DLR Oberpfaffenhofen premises. The Labor transponder was instead placed on a building top in both acquisitions and equipped with patch antennas and horn antennas in the scenes from 1997 and 1998, respectively. The codes used by the Labor and Code 4 transponders were a PN sequence and a Gold code word with a length of 1023 chips, respectively.

The data were first azimuth compressed using a conventional TDC algorithm to verify the correct focusing of the reference nonencoding corner reflectors. Azimuth compression was then repeated using the appropriate code sequence provided by DLR and applying the algorithm described in Section III to focus

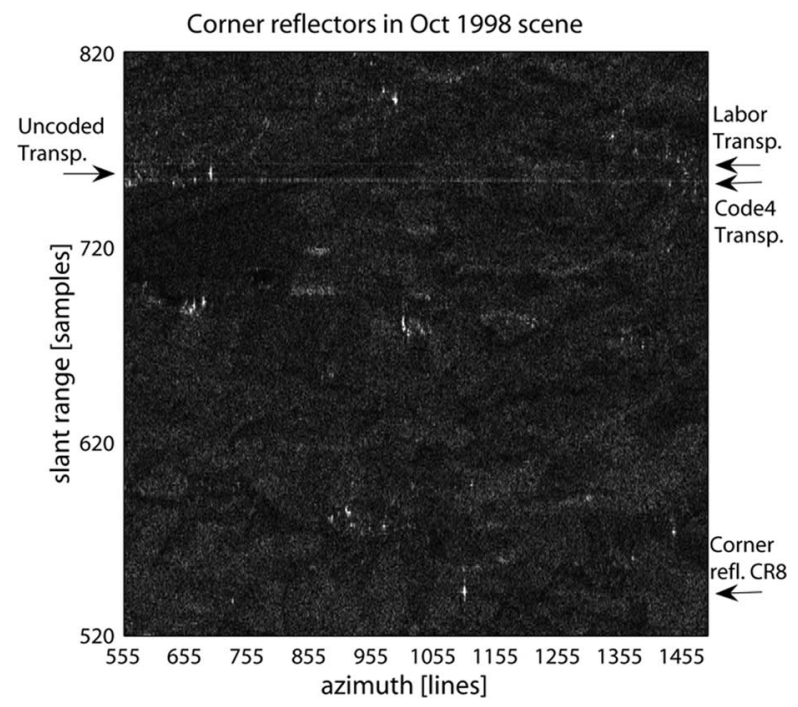

Fig. 6. Corner reflectors in the scene from October 1998 (conventional processing).

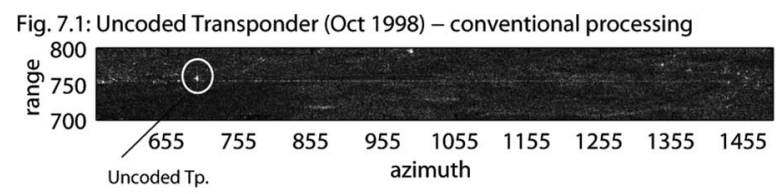

Fig. 7.2: Code4 Transponder (Oct 1998) - processed with code

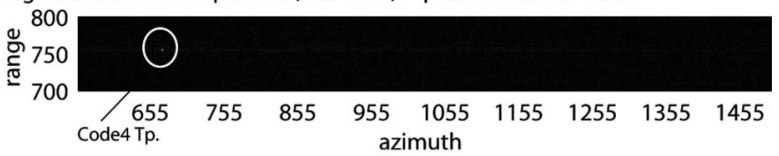

Fig. 7.3: Labor Transponder (Sep 97) - conventional processing

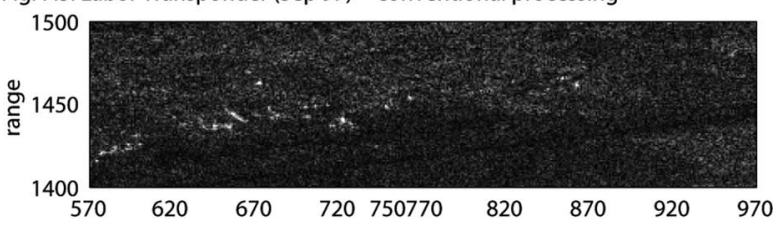

Fig. 7.4: Labor Transponder (Sep 97) - processed with code

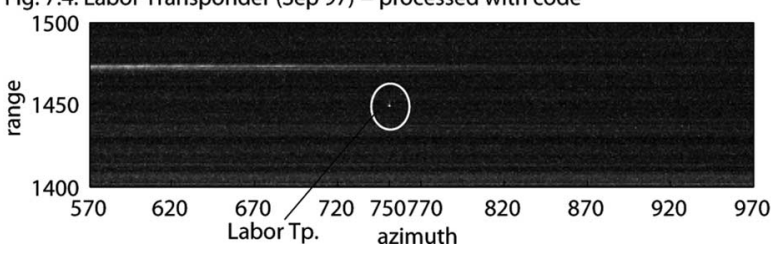

Fig. 7. Matched filtering of encoding and nonencoding point scatterers.

the encoding transponders. Patches of interest of the resulting images are shown in Fig. 7. All point scatterers, and in particular all encoding transponders, were correctly localized. The azimuth positions of the encoding devices in both images were in agreement with the reference ones with an accuracy of less than half a pixel. Horizontal streaks are due to mismatch with the azimuth reference function used, as shown in Fig. 8.

The quality of the focusing was verified by performing vertical and horizontal cuts through the IRF of each focused encoding transponder. The results are listed in Table VI. It can be seen that, when the S/C ratio is high enough to allow measurement of the relevant parameters, the focusing quality of the modified TDC algorithm is the same as that of the conventional TDC algorithm, which in turn meets the expectations for the 


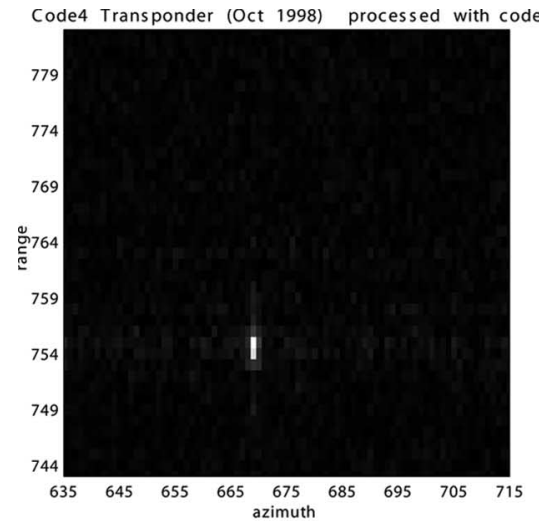

(a)

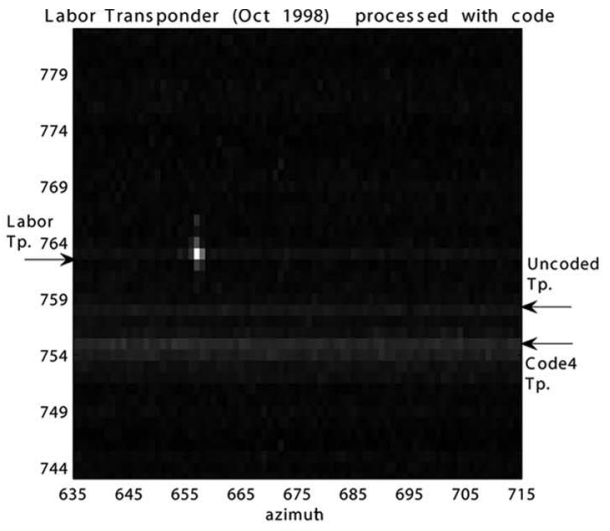

(b)

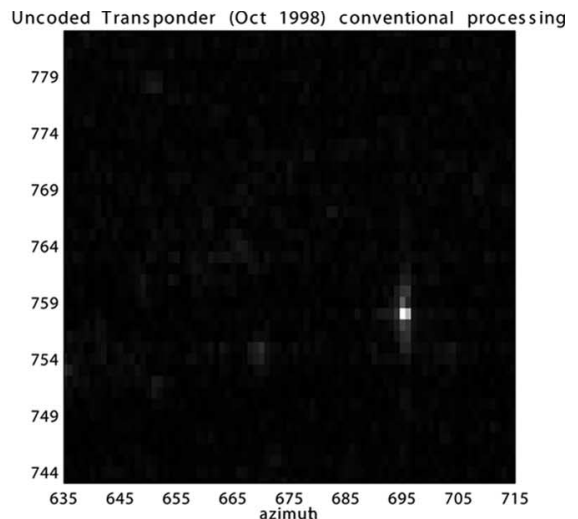

(c)

Fig. 8. Detail of focused point scatterers in the October 1998 scene.

TABLE VI

Point Target IRF Parameters After Matched Filtering

\begin{tabular}{c|cc|cc|cc}
\hline & \multicolumn{2}{|c|}{$\begin{array}{c}-3 \mathrm{~dB} \text { width } \\
\text { slant }\end{array}$} & \multicolumn{2}{c|}{ azimuth } \\
range & (lines) & \multicolumn{2}{c}{$\begin{array}{c}\text { PSLR (dB) } \\
\text { slant }\end{array}$} & azimuth & \multicolumn{2}{c}{ ISLR (dB) } \\
(cells) & & & slant & azimuth \\
range & \\
\hline Uncoded Tp. & 1.15 & 1.34 & -12.05 & -17.4 & -9.17 & -15.26 \\
Lab.Tp.(' 98) & 1.19 & 1.31 & -12.09 & - & - & - \\
Code4 Tp. & 1.19 & 1.34 & -13.11 & -15.69 & -9.57 & -7.47 \\
Lab.Tp.(' 97) & 1.03 & 1.44 & - & - & - & - \\
CR8('97) & 1.16 & 1.31 & -12.03 & -18.96 & -9.44 & -16.11 \\
\hline
\end{tabular}

ERS-2 SAR. An increased ISLR can be noticed, as expected from the simulations presented in Section V.

In order to evaluate the processing gains of Section IV, data processing was directly repeated using the right code alignment for each encoding device, skipping the code acquisition procedure. The peak transponder signal-to-background ratios have been computed by considering a $1-\mathrm{km}^{2}$ area in the upper left corner of Figs. 5 and 6, respectively, as the background, to avoid including the defocused point scatterer signals in the computation of the mean terrain backscattering level. The results are given in Table VII. Keeping the RCS differences into account, gains of 0.97 and $0.4 \mathrm{~dB}$ were observed for the Labor and Code4 transponders, respectively, compared to the Uncoded one. The expected gains according to (9) were 1.15 and $0.88 \mathrm{~dB}$, respectively. In the 1997 scene, the Labor transponder seems to gain $3.55 \mathrm{~dB}$, compared to corner reflector CR8, although it can be seen from Fig. 6 that the two devices are not placed in the same context.

The PBR loss, which can be obtained from Table VII, amounted to 8.5, 8.6, and 9.4 dB for the Code4, Labor' 98 , and Labor' 97 transponders, respectively. The first two values are in excellent agreement with those predicted from the simulations reported in Table $\mathrm{V}$, whereas the third is higher by almost $1 \mathrm{~dB}$. This result might however also be influenced by the low S/C.

Finally, it is possible to infer the peak-to-mean suppression of a nonencoding point scatterer processed with the use of a code sequence. Comparing once again the PBRs of the Labor
TABLE VII

PEAK-TO-BACKGROUND RATIOS

\begin{tabular}{l|c|c|c}
\hline & $\begin{array}{c}\text { observed } \\
\text { (unknown code shift) }\end{array}$ & $\begin{array}{c}\text { observed } \\
\text { (known code shift) }\end{array}$ & $\begin{array}{c}\text { RCS } \\
\left(\mathrm{dBm}^{2}\right)\end{array}$ \\
\hline Uncoded transponder & - & 33.18 & 48.70 \\
Labor transponder (' 98) & 20.90 & 29.49 & 44.04 \\
Code4 transponder & 26.95 & 35.47 & 50.59 \\
CR8 ('97) & - & 33.08 & 50.3 \\
Labor transponder (' 97) & 10.08 & 19.48 & 33.15 \\
\hline
\end{tabular}

and Code4 transponders to that of the Uncoded one in the 1998 scene in Table VII, it can be seen to be about $24 \mathrm{~dB}$ if no code alignment information is known and $33 \mathrm{~dB}$ if it is known in advance, which is in good agreement with the values in Tables III and V.

\section{RELEVANCE FOR THE APPlicATIONS}

The results of the previous sections can be used to investigate the benefit of the considered co/decoding method for radiometric calibration, interferometry, and tagging. For the former two applications, use of nonencoding SAR transponders has been proposed in the literature, and it is of interest to determine whether an additional encoding capability could be exploited. For tagging, the co/decoding method has already been demonstrated as a means of identification [4]. The implementation proposed in this paper has also been proven to be suitable in Section VI. Equations are however required to decide the device RCS and the encoder's code length.

\section{A. Radiometric Calibration and Interferometry}

Applications of reference reflectors to radiometric calibration and interferometry (persistent scatterer and tie-point baseline calibration techniques) rely on the intensity stability and phase stability of the reflector signal, respectively. To this end, the extent of signal decoupling provided by the co/decoding method is relevant, whereas the ability of identifying the reflector is, in general, not required. In Section V-A, it was found that, for the pseudorandom codes considered, the co/decoding method provides a modest $(1 \mathrm{~dB})$ signal decoupling, regardless of code length, compared to natural backscattering. 
Concerning calibration, assuming that the peak method is used for simplicity, the variation coefficient $D_{I}$ of the reference reflector peak intensity is the parameter of interest. This is related to the peak-reflector-signal-to-average-clutter ratio $S_{c}$, which is assumed to be 100 or larger, by [18]

$$
D_{I}=\sqrt{2} S_{c}^{-1 / 2} .
$$

An increment of $d S_{c}$ will therefore cause a relative improvement in $D_{I}$ given by

$$
\frac{d D_{I}}{D_{I}}=-\frac{1}{2} \frac{d S_{c}}{S_{c}} .
$$

For the high $S_{c}$ required for calibration, a 1-dB increase in $S_{c}$ causes an improvement of less than $1 \%$ in terms of amplitude stability $D_{I}$.

As far as interferometry is concerned, the phase standard deviation $\sigma_{\phi}$ of a reflector in a noisy environment can be approximated for high $\mathrm{S} / \mathrm{C}$ ratios $(>12 \mathrm{~dB})$ by the reflector's amplitude variation coefficient $D_{A}$ [19]. Considering a Rice amplitude distribution and using a Gaussian approximation for high $S_{c}$ values, the following can be proven:

$$
\sigma_{\phi} \simeq D_{A} \simeq \frac{1}{\sqrt{2 S_{c}}}
$$

The relative improvement in phase stability due to an increase in $S_{c}$ is again given by

$$
\frac{d \sigma_{\phi}}{\sigma_{\phi}}=-\frac{1}{2} \frac{d S_{c}}{S_{c}}
$$

For a $12-\mathrm{dB} S_{c}$, the co/decoding method would yield a $4 \%$ phase stability improvement.

\section{B. Tagging}

A detection statistic is required first to decide between the absence and presence of an encoding transponder. For each image pixel, the squared and normalized matched filter output can be used, i.e.,

$$
\frac{|g|^{2}}{L_{r} W_{r} L_{\mathrm{az}} W_{\mathrm{az}}}
$$

where $|g|^{2}$ represents the squared modulus of the complex output of the processing algorithm described in Section III, and $L_{r}\left(L_{\mathrm{az}}\right)$ and $W_{r}\left(W_{\mathrm{az}}\right)$ represent the range (azimuth) reference function length and peak amplitude loss due to sidelobe weighting, respectively. Modeling natural backscattering as a complex circular Gaussian random variable and assuming a uniform phase distribution for the tag, the probability density expressions derived in [20] may be used. False alarm rate $P_{\mathrm{FA}}$ determines threshold $\tau$, beyond which the presence of a tag is declared. The probability of detection $P_{D}$ imposes, instead, the minimum detectable tag RCS $\sigma_{\text {tag. }}$. In SAR imagery detection, $P_{\mathrm{FA}}=10^{-8}$ is often chosen. For $P_{D}=0.95$, the following equations were derived in [20]:

$$
\begin{aligned}
\tau & =18.4 \sigma_{\text {clutter }}^{2} \\
\sigma_{\text {tag }}^{2} & \geq 29.3 \sigma_{\text {clutter }}^{2}
\end{aligned}
$$

where $\sigma_{\text {clutter }}^{2}$ represents the residual noise and clutter RCS after matched filtering. Assuming a homogeneous scene, it is given by

$$
\sigma_{\text {clutter }}^{2} \simeq \frac{\left(\sigma^{0}+\mathrm{NE} \sigma^{0}\right) \delta_{\mathrm{gr}} \delta_{\mathrm{az}}}{E\left[G_{d}\right]}
$$

where $\sigma^{0}$ represents the specific backscattering coefficient; $\mathrm{NE} \sigma^{0}$ is the noise-equivalent $\sigma^{0} ; \delta_{\mathrm{gr}}$ and $\delta_{\mathrm{az}}$ are the ground range and azimuth resolutions, respectively; and $E\left[G_{d}\right]$ is the expected improvement in the S/C ratio given in (19). In an operative scenario, $\sigma^{0}$ can be obtained from a conventionally processed and radiometrically calibrated image.

Equation (20), together with (12) (or Table IV), can be used to determine the minimum required code length and tag RCS. Considering ERS parameters, $\mathrm{NE} \sigma^{0}=-21 \mathrm{~dB}$ and $\delta_{\mathrm{gr}}=\delta_{\mathrm{az}}=30 \mathrm{~m}$, and $E\left[G_{d}\right]=1 \mathrm{~dB}$ from Table III. Assuming a representative terrain backscattering of $\sigma^{0}=-10 \mathrm{~dB}$, a $\sigma_{\text {tag }}^{2} \geq 27 \mathrm{dBm}^{2}$ is obtained from (20), a specification that can be met by modern transponders [21]. Code length has an impact on the code synchronization procedure, the success of which ensures the availability of a detection statistic. To this end, from Table IV, it is seen that a code length of 511 chips is the minimum required one. Table IV also indicates how the selection of a higher RCS may allow the use of a shorter code length.

\section{CONCLUSION}

A processing algorithm that is suitable to accurately recover amplitude and phase backscattered from a pulse-to-pulse SAR encoding transponder has been theoretically presented and validated on simulated and real data. A synchronization procedure requiring no a priori information was also described and tested. Its success is related to radar parameters, environment backscattering level, and the length of the encoding sequence. Using a short code sequence reduces the SM for a fixed RCS but, on the other hand, improves the processor's computational efficiency.

As far as interaction with the backscattered field from nonencoding reflectors is concerned, the co/decoding method provides an intensity gain and, thus, a decoupling, compared to a nonencoding point scatterer (or an encoding one using a different code). Such a gain is code length dependent, and its minimum value ranges from about 11 to $22 \mathrm{~dB}$ for the code lengths considered, as detailed in Table III. When placed in a random scene instead, regardless of its statistical properties, signal decoupling is expected to improve only by $1 \mathrm{~dB}$, compared to conventional imaging, although more significant scene-dependent gains might be observed for nonhomogeneous scenes.

For the applications, the co/decoding method with pseudorandom codes is more appealing for its capability to provide reflector identification than for its clutter decoupling properties. Modest benefits were found for radiometric calibration and interferometry in terms of intensity stability and phase stability, respectively, whereas, for tagging applications, the tag RCS and encoder code length specifications were derived and found to be attainable with current systems.

A final remark concerns the extension of the results of the code synchronization and decoupling analysis to other pseudorandom codes besides the Gold ones as PN or Kasami 
sequences. The $\mathrm{S} / \mathrm{C}$ ratio is influenced by the chirp properties and the pseudorandomness of the sequence of phase alterations and the SM by the code's autocorrelation function. Finally, $E\left[G_{c}\right]$ (11) will be influenced by the cross-correlation function between two code words. PN sequences have better autocorrelation properties than Gold codes and are expected to yield a greater SM for a given RCS. Equation (12) with $t(n)=1$ can be used for the computation, yielding approximately a $2-\mathrm{dB}$ improvement. On the other hand, cross-correlation properties are worse, and lower values for $G_{c}$ are expected. In addition, for a given $n$, fewer codes are available. To this end, the large set of Kasami sequences could be used to generate a greater number of different codes for a fixed value of $n$, without worsening the autocorrelation and cross-correlation properties compared to the Gold codes.

\section{ACKNOWLEDGMENT}

The authors would like to thank P. G. Marchetti of the Earth Observation Programme-European Space Agency for his ideas and advice throughout the work; Prof. D. Solimini of the University of Rome "Tor Vergata" for his support; Prof. Dr. A. Moreira, Dr. D. Hounam, and Dr. M. Schwerdt of Institut für Hochfrequenztechnik, DLR, for providing the ERS-SAR data set; D. Manandhar of the University of Tokyo for sharing his knowledge of GPS code acquisition techniques; B. C. Barber of DSTL for the useful discussions on SAR processing issues; and the anonymous reviewers for their interest and constructive comments.

\section{REFERENCES}

[1] H. D. Jackson and A. Woode, "Development of the ERS-1 active radar calibration unit," IEEE Trans. Microw. Theory Tech., vol. 40, no. 6, pp. $1063-$ 1069, Jun. 1992

[2] M. Shimada, H. Oaku, and M. Nakai, "SAR calibration using frequencytunable active radar calibrators," IEEE Trans. Geosci. Remote Sens., vol. 37, no. 1, pp. 564-573, Jan. 1999.

[3] R. Lenz, K. Schuler, M. Younis, and W. Wiesbeck, "TerraSAR-X active radar ground calibrator system," IEEE Aerosp. Electron. Syst. Mag., vol. 21, no. 5, pp. 30-33, May 2006.

[4] D. Hounam and K. H. Wägel, "A technique for the identification and localization of SAR targets using encoding transponders," IEEE Trans. Geosci. Remote Sens., vol. 39, no. 1, pp. 3-7, Jan. 2001.

[5] M. Weiß, "Transponder for calibrating bistatic SAR systems," in Proc. EUSAR, Ulm, Germany, May 25-27, 2004, pp. 925-928.

[6] D. Hounam, "A SAR conjugate mirror," IEEE Geosci. Remote Sens. Lett., vol. 3, no. 3, pp. 373-376, Jul. 2006.

[7] M. Haynes, S. Smart, and A. Smith, "Compact active transponders for operational SAR interferometry applications," in Proc. ENVISAT Symp., Salzburg, Austria, Sep. 6-10, 2004.

[8] P. Bidigare, "The Shannon channel capacity of a radar system," in Proc. Conf. Rec. 36th Asilomar Conf. Signals, Syst., Comput., Nov. 3-6, 2002, vol. 1, pp. 113-117.

[9] I. M. H. Ulander, "Accuracy of using point targets for SAR calibration," IEEE Trans. Aerosp. Electron. Syst., vol. 27, no. 1, pp. 139-148, Jan. 1991.

[10] I. Cumming and F. Wong, Digital Processing of Synthetic Aperture Radar Data. Norwood, MA: Artech House, Jan. 2005.

[11] R. Lenz, J. Pontes, and W. Wiesbeck, "The TerraSAR-X ground calibration system and pattern estimation software," in Proc. IGARSS, Seoul, Korea, Jul. 25-29, 2005, vol. 7, pp. 4894-4897.

[12] J. P. Merryman Boncori, "Implementation of a co/decoding method in SAR processing based on time domain correlation-Preliminary results," in Proc. IGARSS, Anchorage, AK, Sep. 20-24, 2004, vol. 2, pp. $1522-1525$.

[13] B. C. Barber, "Theory of digital imaging from orbital synthetic aperture radar," Int. J. Remote Sens., vol. 6, no. 7, pp. 1009-1057, 1985.
[14] E. H. Dinan and B. Jabbari, "Spreading codes for direct sequence CDMA and wideband CDMA cellular networks," IEEE Commun. Mag., vol. 36, no. 9, pp. 48-54, Sep. 1998.

[15] R. C. Dixon, Spread Spectrum Systems With Commercial Applications. Hoboken, NJ: Wiley, 1994

[16] K. Dumper, C. H. Buck, and A. W. J. Dawkins, "Cost-effective calibration transponders for future synthetic aperture radars," in Proc. IGARSS Hamburg, Germany, Jun. 28-Jul. 2, 1999, vol. 1, pp. 416-418.

[17] K. H. Wägel, D. Hounam, R. Bauer, H. Bloetscher, M. Zink, M. Schwerdt, and B. Mayr, "An encoding SAR-transponder for target identification," in Proc. IGARSS, Hamburg, Germany, Jun. 28-Jul. 2, 1999, vol. 1, pp. 20-22.

[18] A. L. Gray, P. W. Vachon, C. E. Livingstone, and T. I. Lukowski, "Synthetic aperture radar calibration using reference reflectors," IEEE Trans. Geosci. Remote Sens., vol. 28, no. 3, pp. 374-383, May 1990.

[19] A. Ferretti, C. Prati, and F. Rocca, "Permanent scatterers in SAR interferometry," IEEE Trans. Geosci. Remote Sens., vol. 39, no. 1, pp. 8-20, Jan. 2001.

[20] P. Bidigare, T. Stevens, B. Correll, and M. Beauvais, "Minimum radar cross section bounds for passive radar responsive tags," in Proc. 38th Asilomar Conf. Signals, Syst., Comput., 2004, pp. 1441-1445.

[21] D. Hounam, R. Bauer, M. Limbach, P. Sanae, and H. Norman, "A miniaturised coded SAR transponder for target tagging," in Proc. EUSAR, Dresden, Germany, 2006.

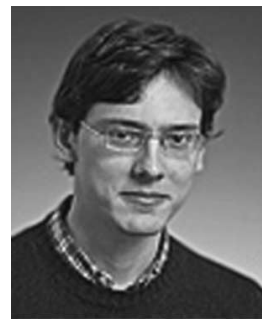

John Peter Merryman Boncori (M'07) was born in London, U.K., on December 2, 1980. He received the Laurea degree (summa cum laude) in communications engineering, carrying out his thesis on encoding SAR transponder signal processing at ESA/ESRIN, and the Ph.D. degree in geoinformation from the University of Rome "Tor Vergata," Rome, Italy, in 2003 and 2007, respectively.

Since January 2006, he has been at Denmark's Technical University, Lyngby, Denmark, as a Visiting Ph.D. student and as a Postdoc, formerly within the Department of Electromagnetic Systems (EMI) and currently within the Danish National Space Center (DRC). His research work here concerned error modeling and correction techniques for SAR interferometry.

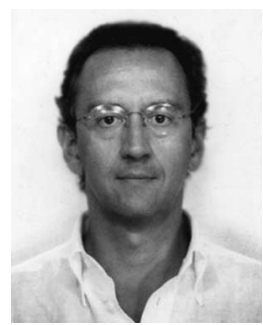

Giovanni Schiavon (M'07) received the Laurea degree (cum laude) in electronic engineering from the University of Rome "La Sapienza," Rome, Italy, in 1982.

$\mathrm{He}$ is currently an Associate Professor with the Dipartimento di Informatica, Sistemi e Produzione, University of Rome "Tor Vergata," where he has been a Researcher from 1984 to 2000 . He has been teaching a course on remote sensing since 1996 and a course on electromagnetic fields since 2000 . He has managed several work packages of different contracts with the ESA. He has been involved in the international remote sensing projects AGRISAR (1986), AGRISCATT (1987 and 1988), MAESTRO-1 (1989), MAC Europe (1991), and SIR-C/X-SAR (1994), and in the ERA-ORA program (European Commission Fourth Framework Programme) on Earth observation for environmental monitoring. He has acted as a Reviewer for the international journals Radio Science and Natural Hazards, and for several volumes of VSP publisher. He has authored or coauthored more than 150 scientific papers, most of which are in international journals or proceedings. His research interests include remote sensing of the atmosphere and of the Earth's surface, propagation, and millimeter waves.

Dr. Schiavon is a Reviewer for the IEEE TRANSACTIONS ON GEOSCIENCE and Remote Sensing and the IEEE Geoscience and Remote Sensing LETTERS. 